\title{
Study of Performance Improvement for Air Shower Array with Surface Water Cherenkov Detectors
}

\author{
A. Shiomi ${ }^{*}{ }^{a}$ K. Hibino, ${ }^{b}$ T. K. Sako, ${ }^{c d}$ T. Asaba, ${ }^{e}$ Y. Katayose ${ }^{e}$ and M. Ohnishi ${ }^{c}$ \\ ${ }^{a}$ College of Industrial Technology, Nihon University, Japan \\ ${ }^{b}$ Faculty of Engineering, Kanagawa University, Japan \\ ${ }^{c}$ Institute for Cosmic Ray Research, University of Tokyo, Japan \\ ${ }^{d}$ Escuela de Ciencias Físicas y Nanotechnología, Yachay Tech, Ecuador \\ ${ }^{e}$ Faculty of Engineering, Yokohama National University, Japan \\ E-mail: shiomi.atsushienihon-u.ac.jp
}

\begin{abstract}
Unlike Imaging Atmospheric Cherenkov Telescopes (IACTs), Extensive Air Shower (EAS) arrays can observe cosmic rays with wide fields of view of about $2 \mathrm{sr}$ and with duty cycles of almost 24 hours every day regardless of weather conditions or the moonlight. Some EAS arrays are located at high altitudes to lower their observation thresholds of cosmic rays and successfully observe gamma rays in the range of several TeV. However, sensitivity of EAS arrays is not as good as IACT mainly because the angular resolution of EAS arrays is poor. In this paper, we report the progress of performance study of an EAS array of scintillation detectors with surface water Cherenkov detectors (WCDs) for improving its angular resolution.
\end{abstract}

35th International Cosmic Ray Conference ICRC2017-

10-20 July, 2017

Bexco, Busan, Korea

\footnotetext{
${ }^{*}$ Speaker.
} 


\section{Introduction}

Cosmic rays up to the knee region are theoretically considered to be accelerated by supernova remnants in our galaxy. Gamma rays from $\pi^{0}$ decays produced by cosmic rays interacting with interstellar matter near the acceleration sites may be observed up to the $100 \mathrm{TeV}$ region. Therefore, observation of $100 \mathrm{TeV}$ region gamma rays is important for searching origins of cosmic rays. As an experiment currently in operation, there is a possibility that a hybrid experiment of an EAS array of scintillation detectors and muon detectors such as the Tibet AS $\gamma$ experiment will open an observation window for the $100 \mathrm{TeV}$ region gamma rays[四. One of the merits of EAS arrays is that they can directly observe the secondary particles regardless of day and night, and can always observe the wide fields of view about 2 sr, which means EAS arrays having such characteristics are more advantageous than IACTs in the low flux of gamma rays expected in $100 \mathrm{TeV}$ region. However, the angular resolution of EAS arrays such as Tibet array is about 0.2 degrees for $100 \mathrm{TeV}$ gamma rays [ [W] , and the angular resolution of $<0.1$ degrees above at $10 \mathrm{TeV}$ or more has not been achieved, so it is a point to be improved. It is also important to be able to investigate more detailed structures of spreading sources.

In order to improve angular resolution, it is necessary to increase the filling ratio of detectors and to determine the shower front more accurately by sampling more time information of secondary particles. By simply placing scintillation detectors at a filling ratio of nearly $10 \%$, it will be considered possible to reach nearly 0.05 degrees at $100 \mathrm{TeV}$ by simulation, but this is costly. WCDs would have ambiguity about particle density and time information as compared to scintillation detectors. However, its effective area is easy to widen, and it is also sensitive not only to secondary electrons but also to secondary gamma rays. Furthermore, since it is possible to increase the filling ratio of detectors at lower cost, it is promising. A scintillation detector capable of accurately measuring secondary particles samples a part of the longitudinal development of extensive air showers, and the accuracy of energy determination can be about $15 \%$ in the $100 \mathrm{TeV}$ region by EAS array [2], and combining the both detectors will make it possible to observe the energy spectrum of gamma rays more accurately. By exploiting the advantages of the both, we will study how far we can improve the angular resolution by experiments of water Cherenkov detectors, based on simulation and prototype detectors.

\section{Simulation}

We have assumed the array of 36 WCDs arranged at $45 \mathrm{~m}$ intervals built at an altitude of $4700 \mathrm{~m}$ a.s.1.. Each WCD consists of a cylinder tank in a diameter of $8.0 \mathrm{~m}$ and variable in height, with a maximum of 13 units, 8 or (virtually) 16 inch PMTs arranged as shown in figure $\square$ and $\rrbracket$. Air showers are generated with the Corsika Ver.7.4 code [3] ], using QGSJET-II-04 and GHEISHA as a hadronic interaction model. Primary gamma rays are vertically generated at $10 \mathrm{TeV}$ aimed to the center of the array. The number of generated air showers is $10^{3}$. After simulating the response of the WCD array for the air showers with the GEANT4.10.02 [四], we obtain the detection efficiency and will obtain the estimated arrival direction in the next step. In this simulation, the maximum quantum efficiency of PMTs is set to $20 \%$. For WCD, it was confirmed that the light intensity reached its maximum at the depth of $1.5 \mathrm{~m}$ (from the top of PMTs). 


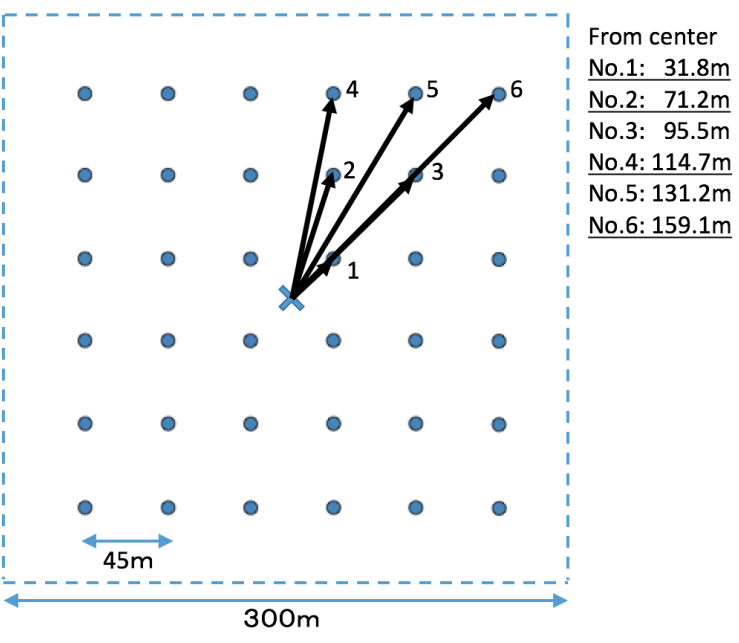

Figure 1: Schematic view of the water Cherenkov detector array. Enclosed by the dashed line is the area of assumed air shower and muon detector array. Gamma rays are vertically injected to the center of the array in this simulation.

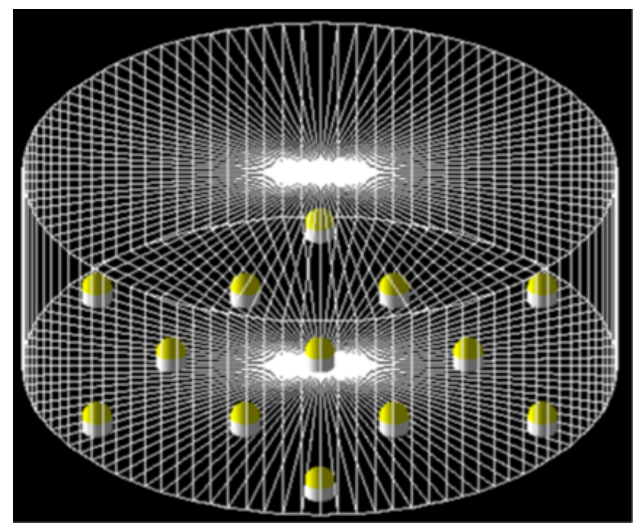

Figure 2: Schematic view of a WCD. WCD is made of a $3.0 \mathrm{~mm}$-thick stainless steel water tank of $8.0 \mathrm{~m}$ diameter. The water level can be changed, and one or 13 upward-facing 8 inch $\phi$ or (virtually) 16 inch $\phi$ PMTs are installed on the floor.

\section{Results and Discussion}

In this simulation, we explain the detection efficiency of WCDs at the depth of $1.1 \mathrm{~m}$ (from the top of PMTs). We investigate the detection efficiency for the case of one PMT in the center, three near the center, seven around the center, and 13 PMTs in the WCD.

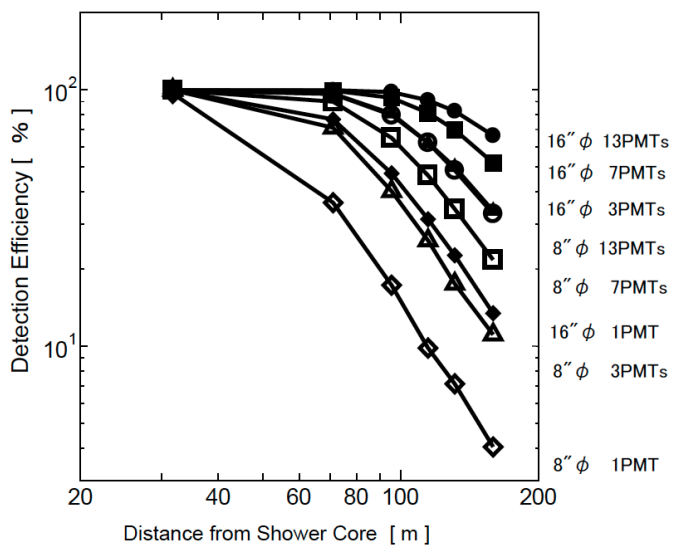

Figure 3: The detection efficiency as a function of the distance between each WCD center and shower core. Trigger condition is any one of PMTs $(\geq 3$ p.e.) in any WCD.

In figure [1, it can be seen that when the distance from the shower core to the center of WCDs is $80 \mathrm{~m}$ or more, the detection efficiency of WCDs is greatly reduced, and it changes with the number and sizes of the PMTs. Figure $\mathbb{\text { Q }}$ shows the detection efficiency as a function of the sum 
of all photosensitive areas of PMTs in a WCD (left) and the ratio of number of triggered WCDs in case of 16" PMTs (right). The trigger condition is any one of PMTs ( $\geq 3$ p.e.) in any WCD. And the case where the trigger condition is any three of PMTs ( $\geq 3$ p.e.) in any one WCD is shown in figure [. It is understood that the detection efficiency correlates with total photosensitive areas in a WCD. Naturally, when the trigger condition is changed from any one to any three, the probability increases as the number of PMTs increases, so the detection efficiency increases as the number of PMTs increases even in the same photosensitive area.

When seven 16 inch PMTs were set in a WCD, it was found that more than 27 WCDs out of 36 detect about $90 \%$ of vertical $10 \mathrm{TeV}$ gamma rays under trigger conditions at any one of PMTs in each WCD. Under trigger condition at any three of PMTs in each WCD, it was found that more than $16 \mathrm{WCDs}$ out of 36 detect about $90 \%$ of vertical $10 \mathrm{TeV}$ gamma rays.
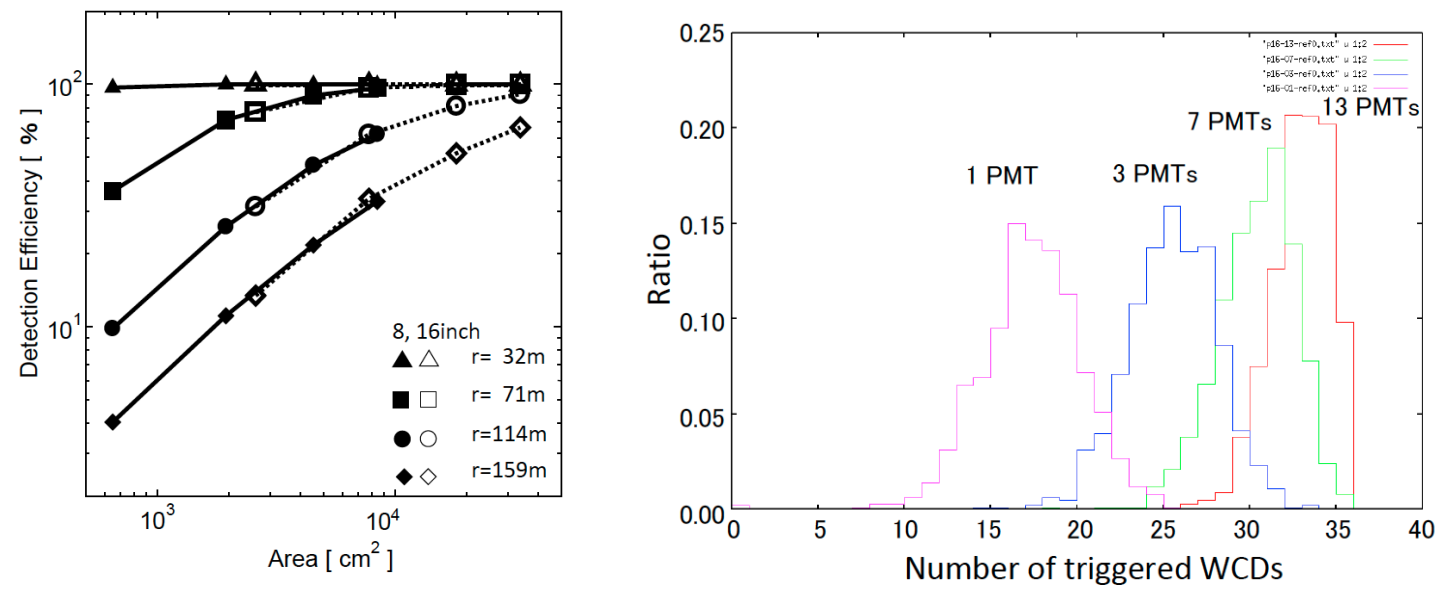

Figure 4: Left: The detection efficiency as a function of the sum of all photosensitive areas of PMTs in a WCD. Right: The ratio of number of triggered WCDs in case of 16 inch PMT. Trigger condition is any one of PMTs $(\geq 3$ p.e. $)$ in any one WCD.
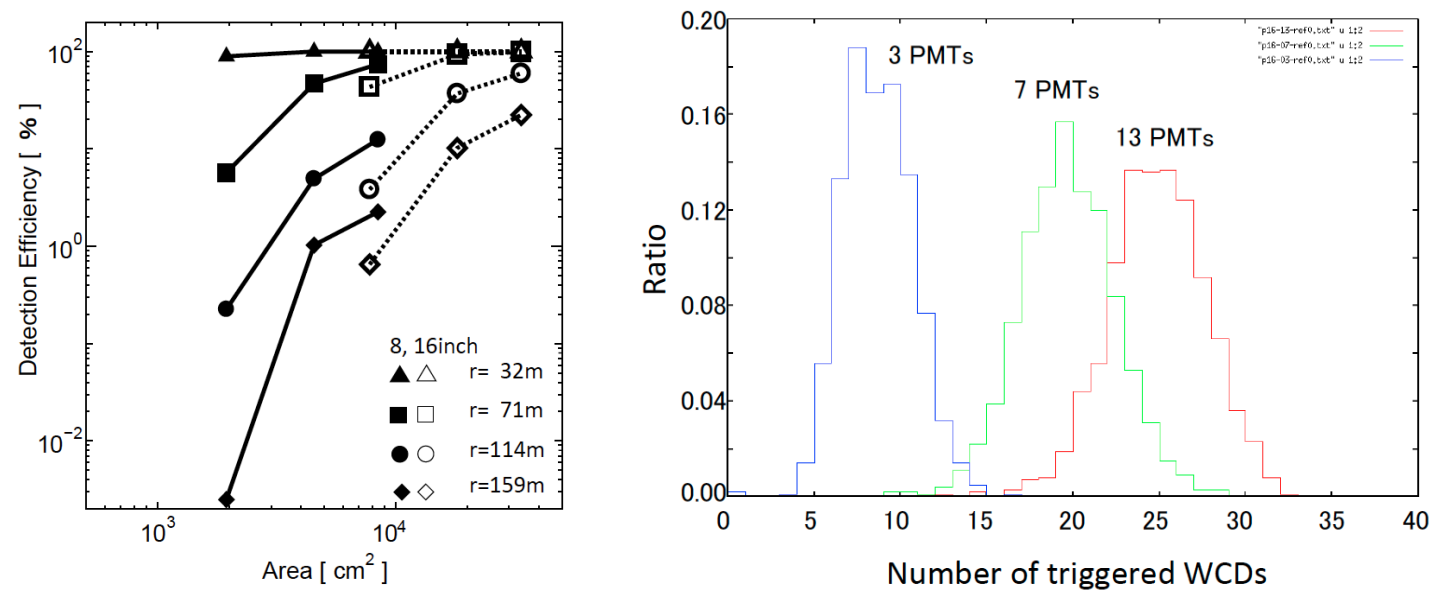

Figure 5: Left: The detection efficiency as a function of the sum of all photosensitive areas of PMTs in a WCD. Right: The ratio of number of triggered WCDs in case of 16 inch PMT. Trigger condition is any three of PMTs ( $\geq 3$ p.e.) in any one WCD. 
In the future, we will investigate the response of a prototype WCD, develop the direction determination algorithm, determine the angular resolution, and explore how angular resolution changes depending on depth, WCD and PMT arrangement, etc.

\section{Acknowledgments}

This work was supported by JSPS KAKENHI Grant Number JP15K05108.

\section{References}

[1] T.K. Sako, et al. Exploration of a $100 \mathrm{TeV}$ gamma-ray northern sky using the Tibet air-shower array combined with an underground water-Cherenkov muon-detector array, Astroparticle Physics, 32177 (2009)

[2] K. Kawata, et al. Energy determination of gamma-ray induced air showers observed by an extensive air shower array, Experimental Astronomy,

[3] D. Heck et al., CORSIKA: A Monte Carlo Code to Simulate Extensive Air Showers, Report FZKA, 6019, Forschungszentrum Karlsruhe (1998)

[4] S. Agostinelli, et al. GEANT4 - a simulation toolkit, Nucl. Instrum. Methods Phys. Res. A, 506250 (2003) 